\title{
Is Early Treatment With Functional Appliances Worth the Effort? A Discussion of the Pros and Cons of Early Interceptive Treatment
}

\author{
Ib Leth Nielsen ${ }^{1, *}$ \\ ${ }^{1}$ Department of Orthodontics, University of California, San Francisco, USA \\ "Corresponding author: Ib Leth Nielsen, Department of Orthodontics, University of California, San Francisco, USA. E-mail: ibortho9@gmail.com
}

Received 2016 April 16; Accepted 2016 May 06.

\begin{abstract}
Early treatment of Class II, Div. 1 malocclusion is a much debated approach and some evidenced based studies using a single appliance has concluded that this approach was mostly not worthwhile. This article will discuss the pros and cons of early treatment with functional appliances and present guidelines for early treatment. The role of facial growth and dentoalveolar development, and growth intensity will be discussed as they relate to treatment outcome.
\end{abstract}

Keywords: Class II, Div. 1, Early Treatment, Functional Appliances, Facial Growth, Treatment Outcome, Retention

\section{Context}

One of the most common malocclusions treated in the orthodontic office is the Class II, Div. 1 that usually is present in combination with a deep bite. The majority of these malocclusions are skeletal and due to an increased sagittal jaw relationship. In the majority of the cases the maxilla in its normal position but the mandible is retrognathic.

Over the years there has been a large number of different functional appliances available to the orthodontist that have been used primarily for treatment in the mixed dentition. These appliances were mostly developed in Europe and became popular primarily because they were removable and therefor did not contribute to further increase in the caries frequency, that in many European countries at the time was a major problem. In the USA these appliances have only to some degree been accepted and never gained the same acceptance as they have in the European countries. In Figure 1 are examples of some of the many different designs that have been used.

There has been much discussion about whether or not the Class II, Div. 1 malocclusion should be treated in the early mixed dentition or later when all teeth have erupted for both financial and practical reasons (Tulloch, 2004) (1). The effectiveness of early treatment has been studies by Wheeler et al. (2) who compared the headgear/biteplate combination to the Bionator. They found that the headgear/biteplate combination was more effective in correcting the Class II malocclusion than the Bionator and that both appliances had a high percentage of the goals achieved. To add to the questions around the best time for treatment, and especially to the issue of treatment timing, or as it has often been presented early versus late treatment, several outcome studies have been conducted. In these studies, the patients were randomly assigned to either a treatment group or a control group and treated with just one appliance. The authors have then concluded that it was not worthwhile to treat early as often a second phase was needed which increased not only the total treatment time but also the cost (Mew) (3).

An interesting issue in this context is that functional appliances have, for many clinicians, disappointingly not been found to increase mandibular growth, and as result orthodontists have more or less discontinued using these appliances. So the picture is in general quite complicated and the results of these cost benefit studies, where functional appliances were used, are questionable. We therefore feel that the topic deserves more attention than it has been given in recent years. In this article we will attempt to clarify some of the issues around early treatment of the Class II, Div.1 malocclusion to hopefully bring some clarification to the topic. It should also be helpful to establish some guidelines for when to treat early and when treatment can be postponed until all teeth have erupted. In these cases of the Class II, Div. 1 malocclusion with deep bite there are several important factors that need to be assessed before any treatment decisions are made. One very important thing to keep in mind is that during the early and late mixed dentition stages the sagittal jaw relationship does not generally change as illustrated in Figure 2. 

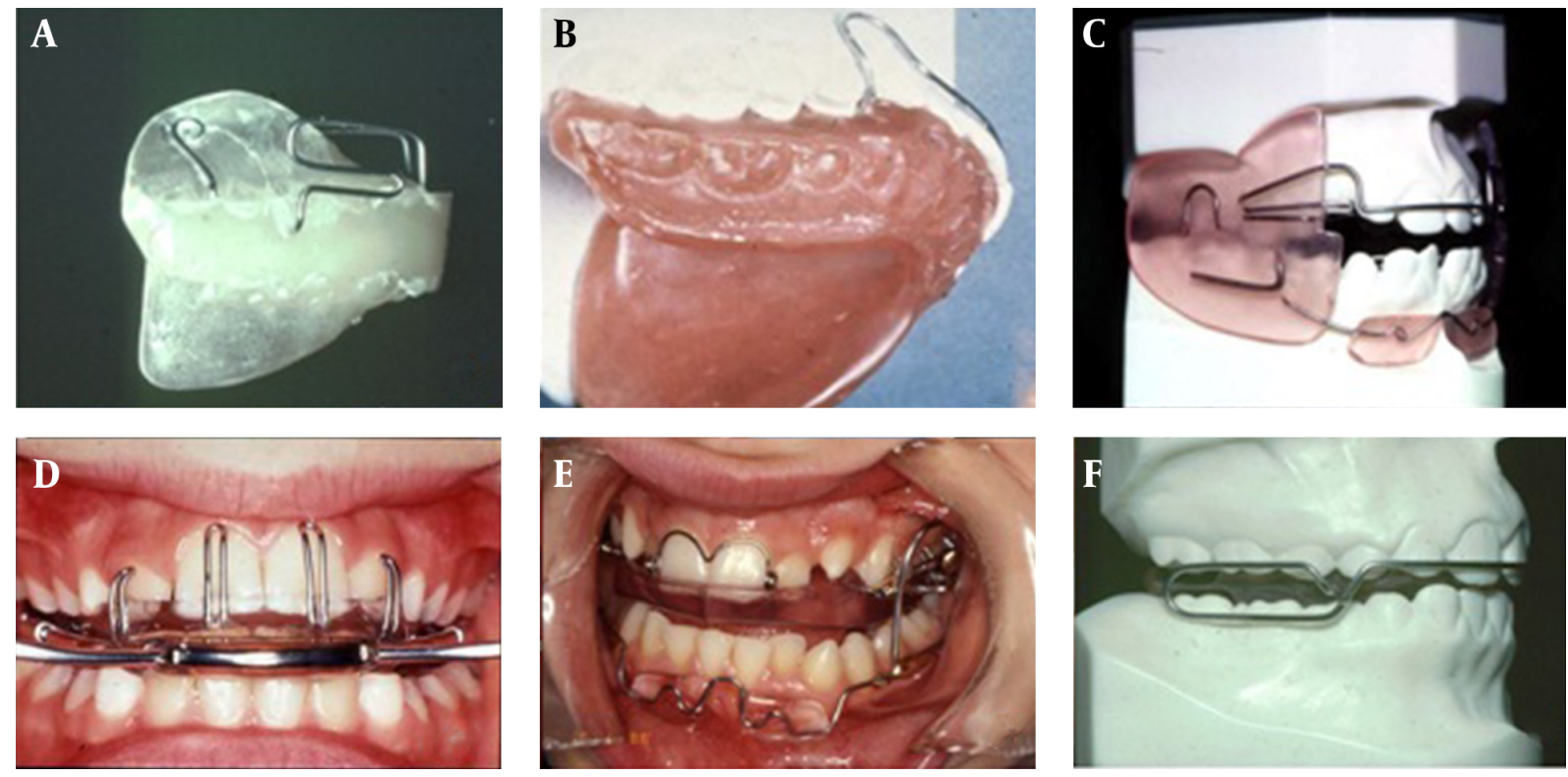

Figure 1. Various Types of Functional Appliances. A, the Harvold Woodside activator; B, the Andresen appliance; C, the Frankel II appliance; D, the Teuscher headgear-activator appliance; E, the bass appliance; F, the Bionator (Balters).

This figure includes 29 subjects with Class II, Div. 1 malocclusion observed over a period of three years without treatment. As seen here the jaw relationship did not improve nor did the overjet and overbite improve. In fact, when looking more closely it can be seen that the maxillary incisors become more proclined thereby increasing the overjet. This increase in proclination is undoubtedly due to a lip dysfunction of the lower lip. The severity of the malocclusion will of course vary from patient to patient, but in the more severe cases, where the overjet is greater than 7 $\mathrm{mm}$ there is a significantly higher risk of traumatic damage to the incisors (Koroluk et al. 2003) which would be a strong indication for early treatment (4).

An additional concern is that a deep bite, often seen in cases with Class II malocclusion and an increased overjet, adds a further challenge to the treatment and may in itself be an indication that early treatment is appropriate. This increase in overbite is mostly the result of overeruption of the lower incisors, as they keep erupting until they make contact with either the palatal mucosa or the gingival tissues behind the upper incisors. This deepening of the bite has been shown to get worse over time and often results in an impinging overbite. This worsening of the bite is caused by the anterior growth rotation of the mandible, that most patients experience during growth (Bjork et al. 1972 ) (5). If, however, the lower incisors during their eruption make contact with the lingual surfaces of the upper incisors there is less of a chance that the overbite will deepen

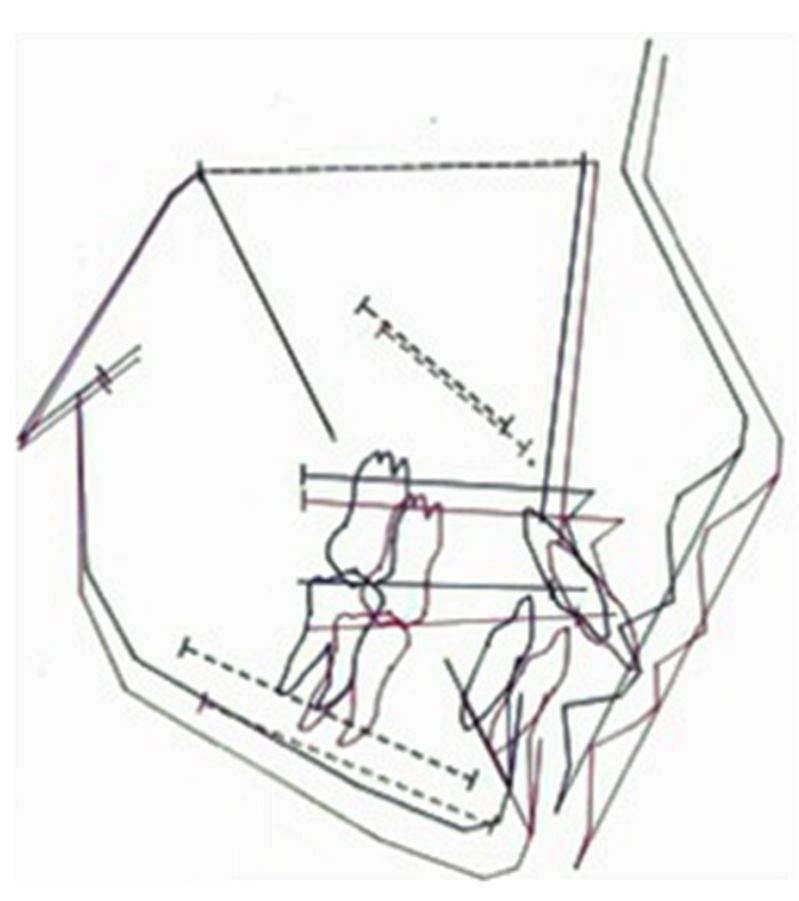

Figure 2. Twenty Nine Subjects With Class II, Div. 1 malocclusion observed over a period of three years without treatment 
during growth, as this now becomes a "fulcrum point" that can resist the effects of the jaw rotation. In those cases, the malocclusion may not need to be corrected early but can be delayed until all teeth have erupted. These are just some of the considerations that may be part of the final decision whether to treat early or later but in individual cases there may be other reasons to consider when deciding on the most appropriate time to treat.

\section{Some Thoughts on Choice Treatment Mechanics}

When looking closely at the illustration, previously shown in Figure 2, one can notice that the maxillary molars migrate mesially during the observation period. This is in part due to forward growth of the maxilla in part to mesial migration within the maxilla. This observation is important as it demonstrates one of the main goals of functional appliance treatment, namely to prevent the normal mesial migration of the upper posterior teeth in addition to restraining forward growth of the maxilla. Additionally, it is one of the goals of treatment to prevent further proclination of the upper incisors during the treatment period. Most of these goals can be achieved by using fixed appliances and headgear, however in patients that are in the early mixed dentition this approach would be limited to a two by four fixed appliance combined with the headgear. However, this approach does not address the lip dysfunction often seen in these patients, and the fixed appliances increase the risk of caries in these young patients. Alternatively, a functional appliance can be used to achieve similar results but with several additional benefits to be discussed further in the following.

An approach that has been popular for many years for early interception of a Class II, Div. 1 malocclusion is to treat the malocclusion with a combination of a bite plate and headgear. The bite plates function is to correct the deep overbite and the cervical headgear to restrain forward growth of the maxilla, hopefully thereby correcting the Class II malocclusion. Although this may work well in cases with good facial growth and a favorable growth pattern, it can also result in downward growth of the mandible in some instances. The example in Figure 3 shows a patient with a Class II, Div. 1 malocclusion, treated with headgear and bite plate, where the mandible didn't growth forward as expected but instead descended vertically, possibly caused by too much tooth eruption relative to the amount of vertical condylar growth during the treatment period. The lack of posterior tooth contact, resulting from the separation of the posterior teeth by the bite plate, permits too much eruption of both the upper and lower posterior teeth. The additional extrusive component of the cervical headgear only adds to the problem with this unintended

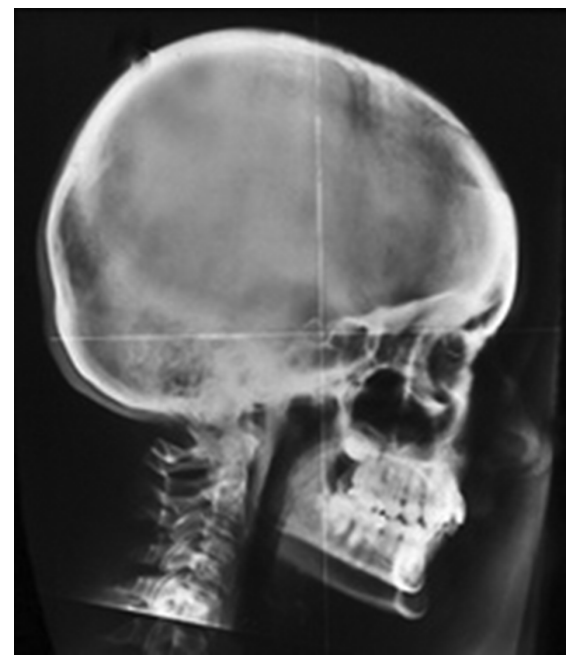

Figure 3. A patient With a Class II, Div. 1 Malocclusion

outcome. As a result of the appliance combination the $\mathrm{AFH}$ (anterior face height) increase in this patient is greater than the increase in PFH (posterior face height). The latter mostly comes from the amount of condylar growth.

The illustration in Figure 4 show the components that make up the AFP and the PFH. The green arrow indicates the condylar growth component that together with lowering of the temporo-mandibular fossa during growth make up the PFH increase. The red arrow represents the sutural lowering of the maxilla that together with the eruption of the maxillary and mandibular molars (blue arrows) make up the AFH change. So in patients where limited growth intensity is expected this combination of appliances, headgear and bite plate, should in general not be used and other approaches with better control of the vertical component of AFH may be more useful.

It is an unfortunate fact that the amount of condylar growth in a juvenile patient in general is unpredictable and that the annual growth of the condyle can vary from as little as $1 \mathrm{~mm}$ to as much as $4-5 \mathrm{~mm}$ per year. The following graphs show these changes in subjects with untreated Class II malocclusion (2).

The amounts of annual condylar growth in girls and boys during the juvenile growth period are seen in Figure 5, (Kim et al. 2002) (6). The subjects all had a Class II malocclusion and did not receive any treatment during the observation period.

The average growth at the condyles is about $3-4 \mathrm{~mm}$ per year, with some individual variations. Additionally the growth intensity fluctuates from year to year in each individual. These facts should be taken into consideration when planning treatment and discussing the estimated 


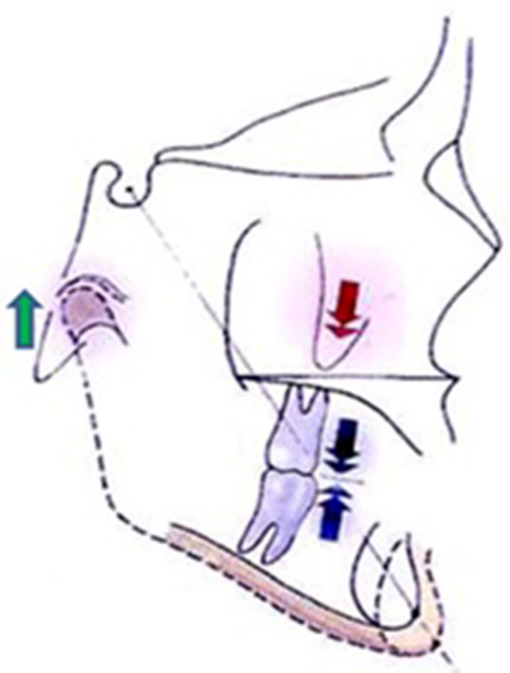

Figure 4. The Components That Make Up the AFP and the PFH

length of treatment with the patient's parents. The limited amount of growth during the juvenile period presents a further challenge namely that it necessitates good vertical control to reduce the eruption of the posterior teeth that otherwise could increase the AFH and prevent forward growth of the mandible.

Functional appliance treatment has been used, as previously mentioned, especially in Europe for many years and quite successfully. It has also to some degree been used in the US with good results, but has lately fallen behind compared to fixed appliance treatment, especially in cases where early-interceptive treatment was indicated. Before we discuss the general function of these appliances it may be a good idea to review the advantages and disadvantages of functional appliances (7). As seen in Figure 6 there are some positive and some negative issues relating to these appliances. A couple of important issues are listed that make these appliances preferable in patients with poor oral hygiene and concerns for caries.

One of the main advantages of functional appliances over fixed appliances is that these appliances are primarily used after school and at night which may be an important consideration in some patients. One clinical advantage, not to be ignored, is that adjustments require very little chair time. An important benefit of functional appliances is that because of the postural position of the mandible they remove any possibility of lip dysfunction while they are in the mouth, which often can be a problem in patients with a large overjet, especially during fixed appliance treatment.

On the negative side it must be mentioned that these appliances do not work well in patients with speech problems and those that are primarily mouth breathers. Also if there is severe crowding of the front teeth it may be more practical to treat with limited fixed appliances, at least initially, before the functional appliance is inserted to correct the skeletal part of the malocclusion.

\section{How do Functional Appliances Work?}

Functional appliances are postural appliances that achieve their correction through muscle function. When the mandible is postured forward, and the patient bites into the appliance, the masticatory muscles are activated and want to retract the mandible back to its normal position. This activation of the muscles is transmitted through the appliances from the mandible to the maxilla and maxillary dentition. The active force results in a combination of tooth movements and skeletal change with a restraining force on the maxilla that restricts its normal downward and forward growth and redirects this growth more vertically. This does not mean that sutural growth of the maxilla is stopped but instead it is changed in direction. The designs of the different functional appliances vary but in general they are constructed to prevent the normal mesial migration of the upper first molars (Figure 2), thereby allowing the mandibular molars to move forward relative to the upper molars with forward growth of the mandible. These appliances in most cases provide some degree of vertical control as there usually is acrylic between the upper and lower posterior teeth. In some instances, however, when there is a deep bite, the acrylic over the lower teeth is removed to allow eruption of the lower posterior teeth so as to correct the overbite.

It is a general misconception that a functional appliance in order to be effective has to provide extra forward growth of the mandible. The vertical and mesio-distal control of the upper posterior teeth and the restriction of maxillary forward growth is in most cases sufficient to correct the Class II and the overjet. It is important, however, to know that there are a number of functional appliances on the market and that they all work pretty much the same way. Some of these are more sophisticated in their design than others, and therefore better suited to minimize undesirable side effects.

Below is a list of some of the side effects to be concerned with. Fortunately, newer designs have managed to get these under better control than in the early days of functional appliances (Figure 7) (7).

Using torque springs against the incisors can prevent the often undesirable retroclination of the maxillary incisors. Our study of the Teuscher Headgear Activator appliance, however showed that the original design was less 
A

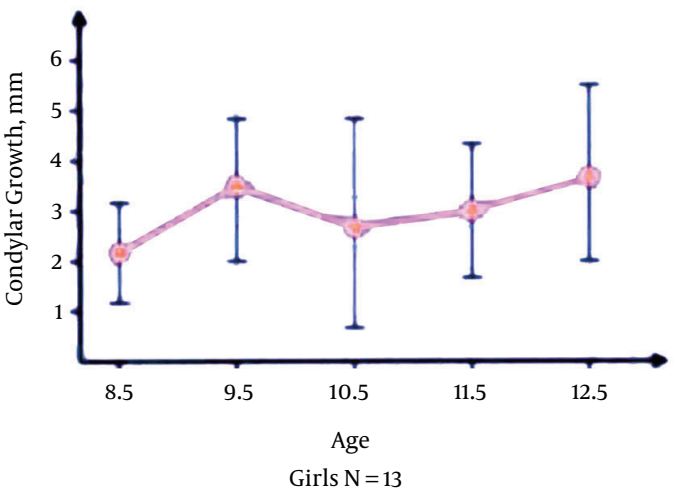

B

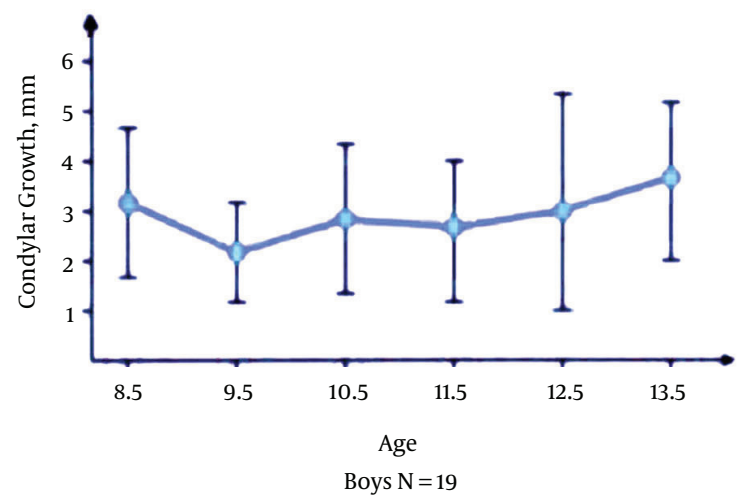

Figure 5. The Amounts of Annual Condylar Growth in A, Girls and B, boys during the juvenile growth period

A

ADVA NTAGES OF FUNCTIONAL APPLIANCES

Provide Instantaneous Improvement in Lip

Function

Minimal Risk of Caries

Can be Used in the Mixed Dentition

Are Primarily Used at Night

Minimal Chair Time for Adjustment
B

DISADVANTAGES OF FUNCTIONAL APPLIANCES

Can Only be Used in Growing Individuals

Limited Control of Individual Teeth

Initial Limited Fixed Appliance May be

Needed

The Appliances are Often Bulky and May

Interfere with Speech

Should Not be Used in Mouth Breathers

Good Patient Cooperation is Needed

Figure 6. A, Some Positive and B, Some Negative Issues Relating to Functional Appliances

A

POSSIBLE SIDE EFFECTS OF FUNCTIONAL APPLIANCES

- Posterior Rotation of the Maxilla

- Posterior Rotation of the Mandible

- Retroclination of Maxillary Incisors

- Proclination of Mandibular Incisors
B

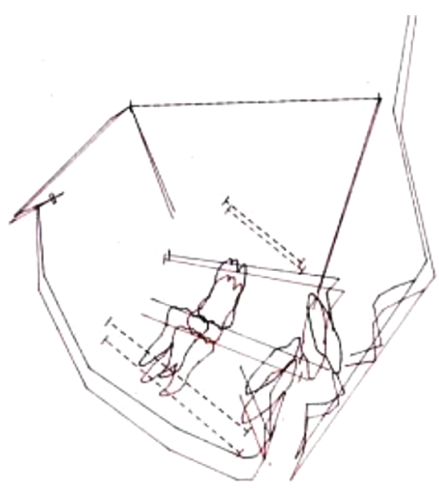


than adequate to control the torque, so they needed to be changed (8). A design by Dr. N. Bass we have found provides adequate control of this torque problem (Figure $8 \mathrm{~A}$ and $8 \mathrm{~B})$.

Any proclination of the lower incisors, that may prevent full correction of the Class II and overjet, can be avoided by capping of the lower incisors. In the Teuscher appliance about $2 \mathrm{~mm}$ of both the upper and lower incisors labial surfaces are covered by acrylic.

Our study of this appliance showed that in a sample of 40 patients the range of variation was \pm 5 degrees (Lagerstrom) (8). We also found when we correlated the initial inclination of the lower incisors with their post treatment inclination that there was an inverse correlation between their initial inclination and the post treatment inclination suggesting that proclined teeth can upright during treatment and retroclined teeth can become more proclined. This finding is contrary to what previous studies have reported, where they concluded that it was a contraindication to use functional appliances in patients with proclined incisors. The posterior rotation tendency, as measured by the mandibular plane angle, that has been reported with functional appliances, we found did not happen in our study. Correlating the pre-treatment mandibular inclination with post treatment showed no significant change. A summary of the linear and angular changes of the incisors in 40 patients during treatment with the Teuscher appliance is seen in Figure 9. Note the amount of forward growth of the mandible in this 18 months' period and the lack of mesial migration of the upper molars. It is also important to notice that the maxillary incisors despite the use of torque springs became more retroclined during treatment, whereas the lower incisors on average maintained their inclination.

\section{Setting Up the Occlusion}

An interesting aspect of the studies that looked at the cost benefit of early treatment was that it was of little or no benefit as discussed earlier in this article. It should be mentioned that in those studies there was no initial preparation of the dental arches prior to the insertion of the functional appliance. This is a different protocol from what has been used in Europe for instance where the dental arches nearly always are set up with initial maxillary expansion, retroclination of the lower incisors and proclination of the upper incisors. This is to prepare the arches for the functional appliance so that full correction of the molar occlusion can take place and to ensure adequate arch width that will make it possible to finish with a good occlusion in all three planes of space. When this is not done the correction can only be partially successful and the patient is often left with a half cusp Class II malocclusion and a partially corrected overbite and no overjet, making a second phase of treatment necessary. Two such appliances are seen in Figure 10.

The appliances seen in Figure 10 is a maxillary Hawley retainer with an expansion screw to increase the maxillary dental arch width. There are also two overlapping finger springs to permit proclination of the maxillary incisors if needed. Finally, the appliance has a bite plane to assist in correction of the overbite. These appliances have an additional purpose namely that they work as "training devices." Using a retainer first tends to increase the cooperation of the patient later when the functional appliance is to be used as for most patients it makes it easier to get used to the larger functional appliance.

\section{Choosing the Right Appliance}

The choice of appliance for early treatment should be dictated by the need for control of the factors listed earlier in Figure 7. If it is important to prevent retroclination of the maxillary incisors then torque springs can be added, if not a conventional labial bow may be the right choice. Having a headgear as part of the appliance, as in the Teuscher appliance, has the additional advantage that it helps the patient keep the appliance in place and connected to the upper arch, which can be a problem if the patient sleeps with the mouth open. The combination of the high-pull headgear and activator further acts as a splint headgear should the patient not bite into the appliance during sleep. Other functional appliance without headgear do not have this advantage and the postural effect, that activates the masticatory muscles, is therefore not present when the patient does not bite into the appliance. As mentioned previously, these appliances in general can be used in the mixed dentition because they can be adjusted when deciduous teeth are lost. An added benefit of early treatment is that patients in the mixed dentition often are more cooperative than later and this gives these appliances a better chance to work.

It is of course very important to monitor the progress of ongoing treatment which can be done by measuring the overjet at each visit. One should expect a $2-3 \mathrm{~mm}$ decrease over the first $6-8$ weeks which is not uncommon. After that 1-2 mm per month can be expected, however, if there is no change between visits the cooperation should be reviewed with the patient and the parents. This change in overjet is usually a combination of dento-alveolar change and growth changes. 

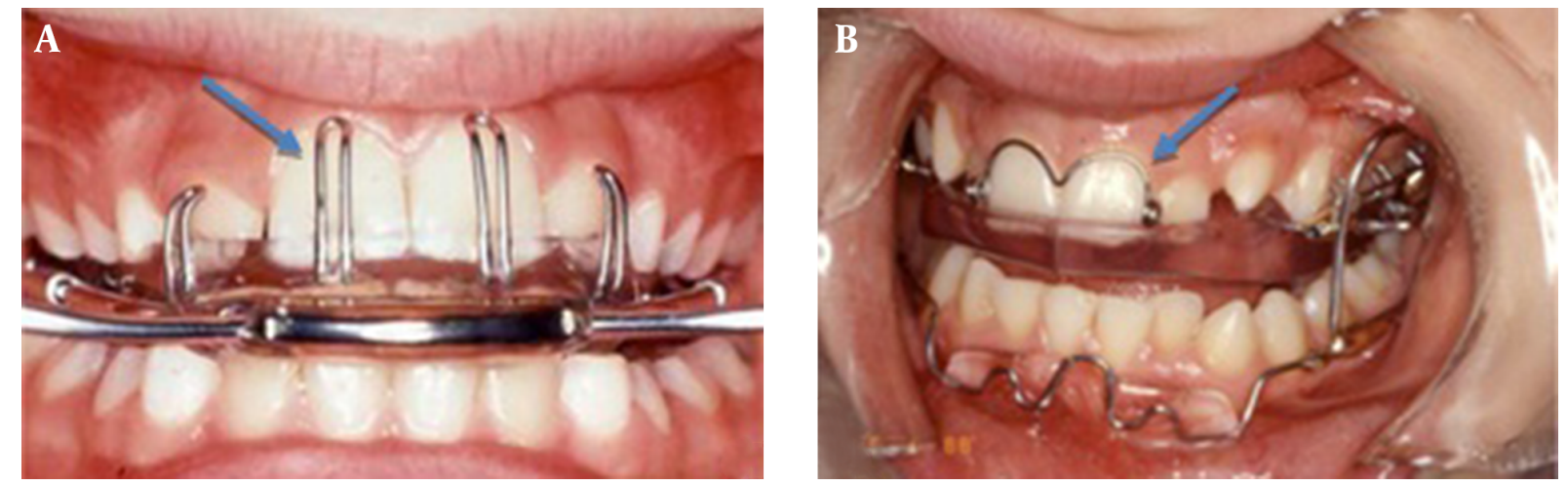

Figure 8. A, The Teuscher Appliance; B, The Bass Appliance

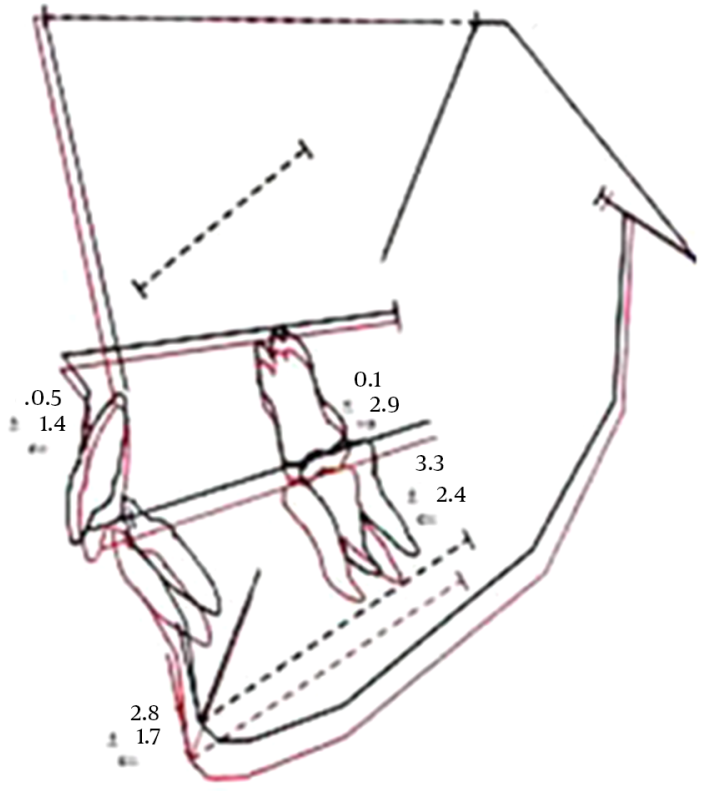

Figure 9. Mean Facial Growth in Patients Treated with The Teuscher Applicance ( $\mathrm{n}=$ 40)

\section{Treatment With the Teuscher Appliance}

In Figure 11 is an example of early treatment with a Teuscher appliance that demonstrates the effectiveness of this appliance combination in a patient in the early mixed dentition.

\section{Retention}

When the occlusion, the overjet and deep bite have been corrected it is important not to stop treatment right away, but to maintain the functional appliance use at night to avoid relapse, this is a point that is often overlooked, when it seems the initial malocclusion is corrected. A minimum of six months is in most cases needed to ensure a stable occlusion. It is also important to make sure the patient is not posturing forward as extended use of a functional appliance can promote this tendency due to muscle conditioning from using the appliance over a period of time.

\section{Conclusion}

In conclusion it can be said that the Class II, Div. 1 malocclusion combined with deep bite can successfully be treated in the early mixed dentition with functional appliances. Timing of treatment should be dictated by the extent of overjet and overbite so that milder cases can be delayed until most permanent teeth have erupted, whereas severe case should be intercepted early. The type of functional appliance used should be dictated by the need to control the factors that can interfere with correction of the occlusion. Transverse and vertical correction with either a retainer or limited fixed appliance should in most cases precede treatment with the functional appliance in order to allow maximum occlusal correction. 

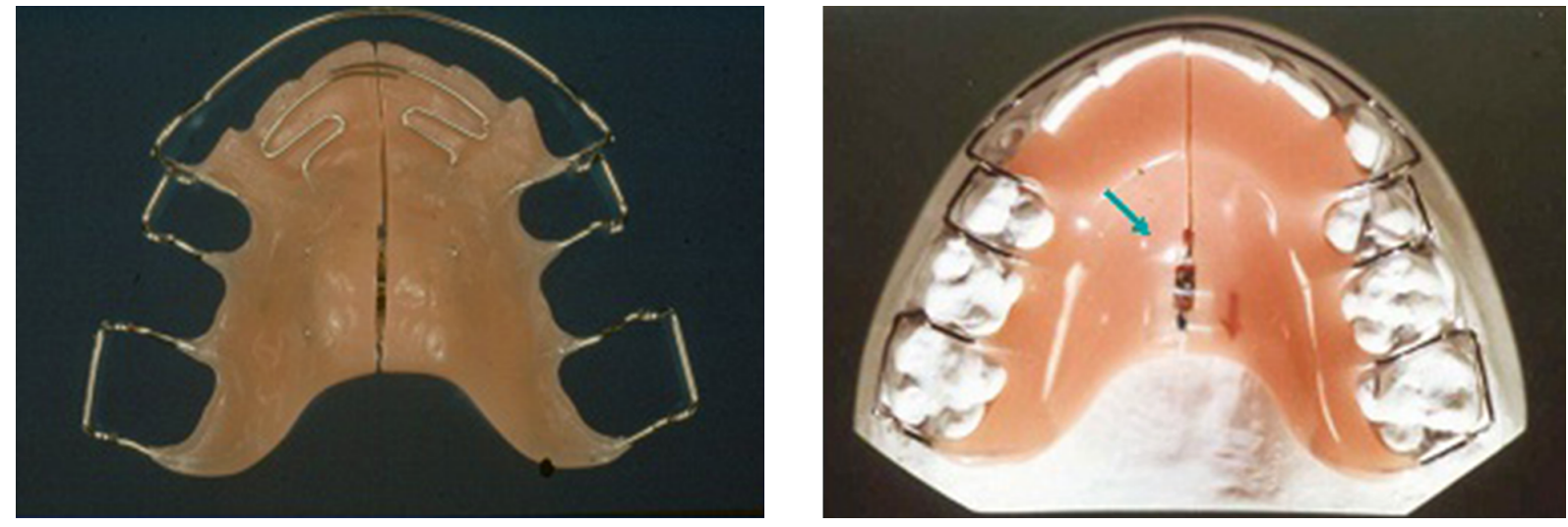

Figure 10. A, Permits Transverse and Sagittal Expansion of the Maxillary Dental Arch; B, Bite Plane to Correct deep Overbite (Transverse Expansion 1 - 2 Turns a Week)
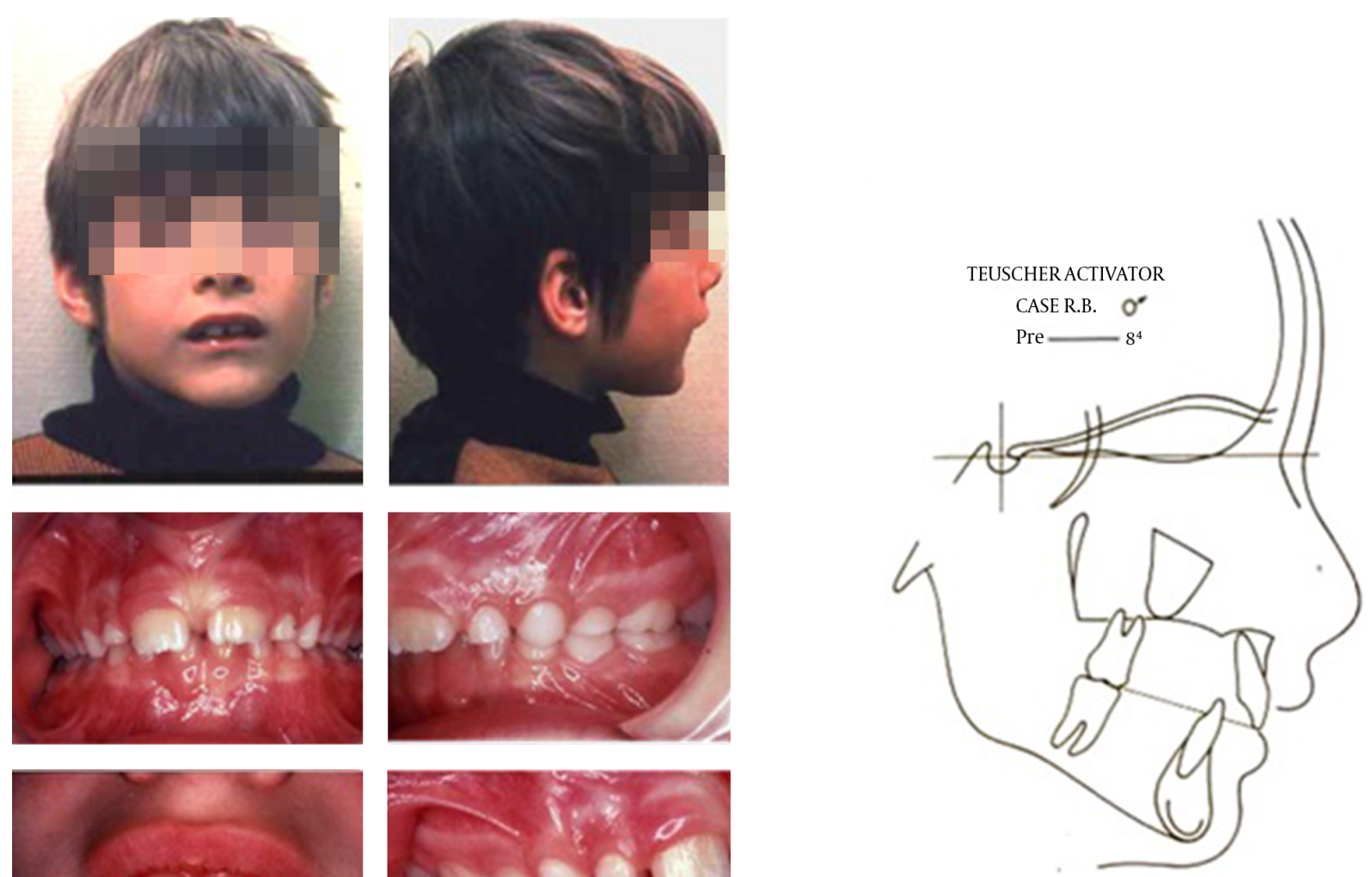

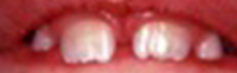

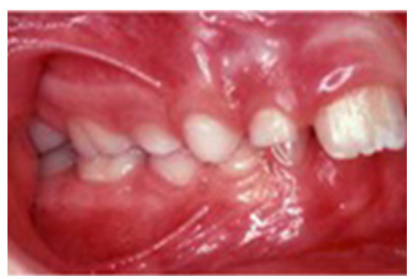

Figure 11. Pretreatment Photos and Cephalometric Tracing of an 8 yr. 4 Mos, Old boy with a Class II, Div. 1 malocclusion and a deep overbite. Note the position of the lower lip. 

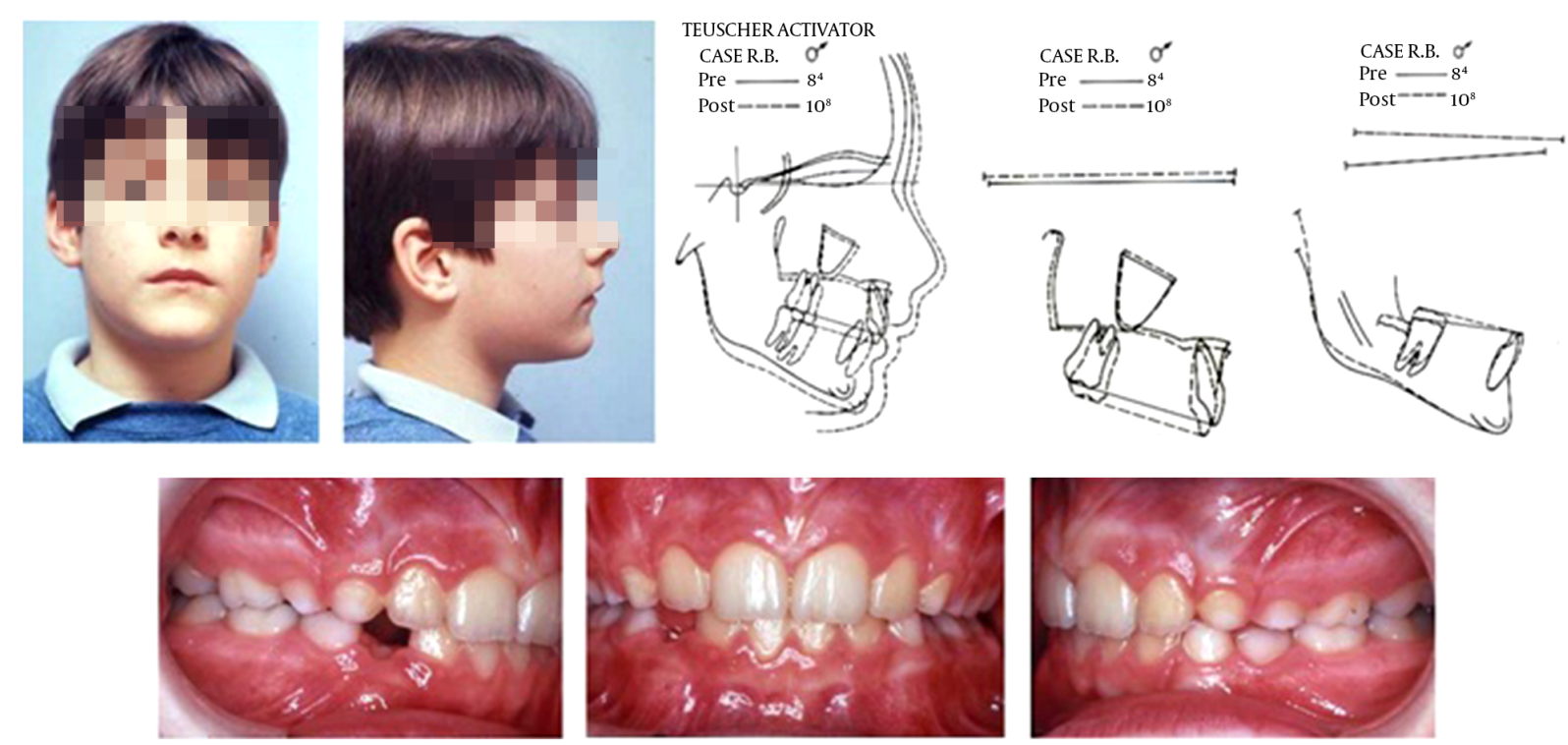

Figure 12. Post Treatment Photos and Superimpositions After Treatment With the Teuscher Appliance, Treatment time 2 yrs. 4 mos.

It seems clear from the available data that in cases with excessive overjet and a deep overbite it is indicated to treat the malocclusion in the early mixed dentition to prevent permanent damage to the soft tissues in the palate by the deep bite and reducing the risk for trauma to the maxillary incisors. Where the Class II is more moderate and the overjet less than $6 \mathrm{~mm}$, and the deep bite is not impinging on the palatal tissues it may be reasonable to delay onset of treatment until the permanent dentition.

\section{References}

1. Tulloch JF, Proffit WR, Phillips C. Outcomes in a 2-phase randomized clinical trial of early Class II treatment. Am J Orthod Dentofacial Orthop. 2004;125(6):657-67. doi: 10.1016/S088954060400160X. [PubMed: 15179390].

2. Wheeler TT, McGorray SP, Dolce C, Taylor MG, King GJ. Effectiveness of early treatment of Class II malocclusion. Am J Orthod Dentofacial Orthop. 2002;121(1):9-17. [PubMed: 11786865].
3. Mew J. The dilemma of early treatment. Am J Orthod Dentofacial Orthop. 2008;133(6):784-5. doi: 10.1016/j.ajodo.2008.04.005. [PubMed: 18538228] discussion 785-6.

4. Koroluk LD, Tulloch JF, Phillips C. Incisor trauma and early treatment for Class II Division 1 malocclusion. Am J Orthod Dentofacial Orthop. 2003;123(2):117-25. doi: 10.1067/mod.2003.86. [PubMed: 12594415].

5. Bjork A, Skieller V. Facial development and tooth eruption. An implant study at the age of puberty. Am J Orthod. 1972;62(4):339-83. [PubMed: 4506491].

6. Kim J, Nielsen IL. A longitudinal study of condylar growth and mandibular rotation in untreated subjects with class II malocclusion. Angle Orthod. 2002;72(2):105-11. doi: 10.1043/00033219(2002)072<0105:ALSOCG>2.0.CO;2. [PubMed: 11999932].

7. Nielsen IL. Guiding occlusal development with functional appliances. Aust Orthod J. 1996;14(3):133-42. [PubMed: 9528411].

8. Lagerstrom LO, Nielsen IL, Lee R, Isaacson RJ. Dental and skeletal contributions to occlusal correction in patients treated with the highpull headgear-activator combination. Am J Orthod Dentofacial Orthop. 1990;97(6):495-504. doi: 10.1016/S0889-5406(05)80030-7. [PubMed: 2353679]. 